\title{
Erratum zu: Die eindeutige Primfaktorzerlegung
}

\section{Urs Stammbach}

Angenommen: 4. Dezember 2009 / Online: 20. Januar 2010

(C) Springer-Verlag 2010

\section{Erratum zu: Math Semesterber 10.1007/s00591-008-0050-6}

Der erste Abschnitt auf Seite 108 oben enthält Fehler. Er ist zu ersetzen durch das Folgende:

Wir werden in diesem Beitrag nur Unterringe $R$ von komplexen Zahlen betrachten, deren additive Gruppe frei abelsch von endlichem Rang ist. Derartige Ringe sind nullteilerfrei und Noethersch. Deshalb gilt, dass eine Nichteinheit immer eine Zerlegung in ein Produkt von unzerlegbaren Faktoren besitzt (siehe z. B. [1], S. 200). Die Frage stellt sich also in solchen Ringen in einer etwas anderen Form, nämlich ob derartige Faktorzerlegungen in unzerlegbare Elemente (bis auf Einheiten) eindeutig sind.

\section{Literatur}

1. Zariski, O., Samuel, P.: Commutative Algebra, vol. I. Van Nostrand, New York (1958)

The online version of the original article can be found under doi: 10.1007/s00591-008-0050-6

U. Stammbach $(\bowtie)$

Mathematik, ETH-Zentrum, 8092 Zürich, Switzerland

E-Mail: stammb@math.ethz.ch 